\title{
Reduction of copper oxide powder by an inductively coupled thermal plasma
}

\author{
H Bissett, M Makhofane, S Lötter \\ Applied Chemistry, South African Nuclear Energy Corporation SOC Ltd. (Necsa) Pretoria, South Africa \\ Email: milton.makhofane@necsa.co.za
}

\begin{abstract}
Additive manufacturing (AM) methods can be utilised to manufacture complex, custom Ti6Al4V components for medical implants. Infection at the bone-implant interface is a key reason for implant rejection. Advanced titanium implants with biocompatibility and antibacterial properties can be manufactured by modifying the titanium alloy with copper, which in small concentrations $(<1$ at $\%$ copper) is a proven, non-toxic antibacterial agent. Copper can be embedded into the titanium implant during the AM process creating antibacterial functionality. In order to produce sufficiently fine metallic copper powder, copper oxide can be reduced, either by chemical reduction or thermal treatment methods. These include thermal decomposition or reduction of the oxide in the presence of a reactive gas at elevated temperatures. Making use of thermal treatment methods such as thermal plasma reduction, the process conditions can be tuned to manipulate the morphology and average particle size of the powders. The purpose of this study was to investigate the thermal plasma reduction of copper oxide to copper metal making use of the Tek-15 radio-frequency inductively coupled thermal plasma system at Necsa.

In the presence of hydrogen, the black copper (II) oxide powder was converted to a dark red powder, while a yellow / orange coloured powder was obtained without hydrogen being present. A change in composition was observed using SEM-EDS and was confirmed by XRD analysis.
\end{abstract}

Keywords: Copper, Thermal Plasma, Reduction.

\section{Introduction}

Initially termed rapid prototyping (RP), Additive Manufacturing (AM) is a layer-by layer production technology that enables the "printing" of three dimensional objects on demand. ${ }^{1}$ It is seen as part of the 4th industrial revolution. AM enables more cost and resource efficient small-scale production. ${ }^{2}$ AM technologies are applied in various industries such as aerospace, where a small number of complex components are required, and the medical sector for the manufacturing of highly personalised one-off products. ${ }^{1}$ The value of aluminium and vanadium as alloy additions was established in the "Workhorse alloy" Ti6Al4V (Ti64), which was patented by Crucible Steel in 1954. Since then, Ti64 has been used for lightweight parts in various industries especially in the medical sector for personalised implants, offering good balance in strength, ductility, fatigue and fracture properties. ${ }^{3}$ However, infection at the bone-implant interface can cause implant failure directly after implantation and for this reason the utilisation of materials with embedded antibacterial properties can be advantageous. ${ }^{4}$

Copper and zinc have potent antibacterial effects on a wide spectrum of bacterial species, but can be toxic to humans at high concentrations. $^{5}$ Therefore, advanced titanium implants with biocompatibility and antibacterial properties can be manufactured by modifying the titanium alloy with copper, which in small concentrations ( $<1$ at $\%$ copper) is a proven non-toxic antibacterial agent. By addition of pure copper powder during the manufacturing process, the copper can be embedded into the titanium implant creating antibacterial functionality. ${ }^{6}$ If, however, the particle size of the copper powder is too large, segregation at fusion boundaries can occur during in-situ alloying where a component is manufactured by laser powder bed fusion. A fine copper powder might therefore be required. ${ }^{7}$

The synthesis of copper metal from copper oxide can largely be achieved by either chemical reduction ${ }^{8,9}$ or thermal treatment methods which include thermal decomposition or reduction of the oxide in the presence of a reactive gas at elevated temperatures. The reduction occurs in the sequence $\mathrm{CuO} \rightarrow \mathrm{Cu}_{4} \mathrm{O}_{3} \rightarrow \mathrm{Cu}_{2} \mathrm{O} \rightarrow \mathrm{Cu}$, in which $\mathrm{Cu}_{4} \mathrm{O}_{3}$ is a metastable state. Although hydrogen $\left(\mathrm{H}_{2}\right)$ is a good reductant, $\mathrm{H}$ and $\mathrm{H}^{+}$species present in a hydrogen plasma are much stronger reductants and therefore a plasma process will lower the activation energy required for reduction of copper(II) oxide to copper. ${ }^{10}$ The purpose of this study was therefore to produce copper metal by the reduction of copper oxide in a thermal plasma.

\section{Material and methods}

\subsection{Experimental set-up}

In all experiments the Tek-15 thermal plasma system was used. The basic radio frequency (RF) torch design is shown in Figure 1. The powder was fed through the feeding probe with the assistance of a carrier gas. The powder was rapidly heat treated and reacted in the plasma "tail flame". A quartz tube separated the sheath and central gas (Ar), both of which could be adjusted to alter the plasma parameters. 


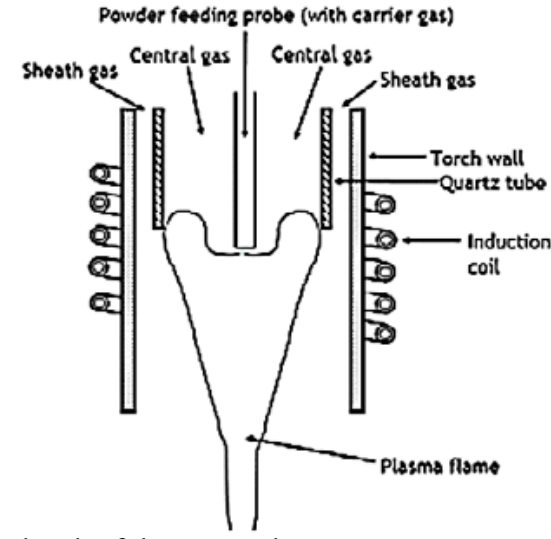

Figure 1: Sketch of the RF torch

\subsection{Materials and parameters}

In order to evaluate whether copper(II) oxide can be reduce to copper powder in a thermal plasma, fine copper(II) oxide powder (Merck CAS \# 1317-38-0) was used as starting material (feed powder). Two experiments were conducted; with and without hydrogen gas using an argon plasma. The experimental parameters are shown in Table 1. The powder was introduced into the plasma by means of a carrier gas.

Table 1: Copper(II) oxide powder treatment at various plasma conditions. Experiments performed at $85 \mathrm{kPa}$ reactor pressure, central gas flow of $10 \mathrm{slpm}$ and carrier gas flow of $2 \mathrm{slpm}$

\begin{tabular}{ccc}
\hline Plasma plate power & $\mathbf{H}_{\mathbf{2}}$ in sheath gas & Powder feed rate \\
\hline $\mathbf{( k W )}$ & $\mathbf{( \mathbf { v } / \mathbf { v } \% )}$ & $\mathbf{( k g / h )}$ \\
\hline 11 & 0 & 0.24 \\
12 & 5 & 0.15 \\
\hline
\end{tabular}

${ }^{*}$ slpm $=$ standard litres per minute

\subsection{Powder characterisation}

Powder phase identification was obtained by X-ray diffraction technique (XRD), in conjunction with 2020 PDF-4+ database making use of a Bruker XRD D8 Advance with a copper $\mathrm{K} \alpha$ radiation source $\lambda=0.15406 \mathrm{~nm}$.

The copper(II) oxide feed and plasma treated powders were submitted for SEM EDS analyses performed using a FEI Quanta 3D Dual Beam SEM with an EDAX Octane Elect SD detector running the TEAM ${ }^{\mathrm{TM}}$ EDS Analysis System to determine if a change in chemical composition before and after plasma treatment had occurred. Rietveld refinement was applied to determine the phase composition of the powders.

\subsection{Thermodynamic calculations}

The equilibrium module of HSC Chemistry 6 software was used to study the reduction of copper(II) oxide to copper metal with and without hydrogen present. The program calculates the equilibrium composition using the Gibbs solver, which uses the Gibbs energy minimization method. Equation 1 was used to calculate the Gibbs free energy at any condition. At equilibrium the Gibbs free energy is equal to zero and Equation 2 can be used to predict the equilibrium composition.

$$
\Delta G=\Delta G^{0}+R T \ln k
$$

At equilibrium $\Delta G=0$ :

$$
\Delta G^{0}=-R T \ln k
$$

where $\Delta G$ is Gibbs free energy at any condition, $\Delta G^{0}$ is the Gibbs free energy at standard conditions, $\mathrm{R}$ is the universal gas constant, $\mathrm{T}$ is the temperature and $\mathrm{k}$ is the equilibrium constant.

\section{Results and discussion}

Copper(II) oxide $(\mathrm{CuO})$ is a black powder, while copper(I) oxide $\left(\mathrm{Cu}_{2} \mathrm{O}\right)$ is a red-brown powder and pure copper $(\mathrm{Cu})$ a red powder. The colour of copper oxide powders is, however, also dependent on the powder particle size and the colour observed can vary from yellow to dark red. ${ }^{11}$ The powder collected after treatment at $11 \mathrm{~kW}$ in argon only had a dark red colour, which was probably copper(I) oxide. The powder obtained after treatment at $12 \mathrm{~kW}$ with hydrogen was a yellow to red coloured powder, which can be either copper metal, copper(I) oxide, or a mixture of the two. In Figure 2 the XRD phase spectra of the powders before and after plasma treatment is shown. It was clear that a change in phase composition had occurred after plasma treatment with and without the addition of hydrogen.

In Table 2 it can be seen that the feed powder was pure copper(II) oxide containing no other phases. The treatment of the copper(II) oxide powder at $11 \mathrm{~kW}$ in argon only converted the majority of copper(II) oxide $(\mathrm{CuO})$ to copper(I) oxide $\left(\mathrm{Cu}_{2} \mathrm{O}\right)$, while some copper metal $(\mathrm{Cu})$ was obtained. It was interesting to observe that the treatment at $12 \mathrm{~kW}$ in the presence of hydrogen resulted in the direct conversion to copper metal powder, with no copper(I) oxide $\mathrm{Cu}_{2} \mathrm{O}$ formation obtained, while leaving some copper(II) oxide $\mathrm{CuO}$ unconverted. The temperature profile of the plasma caused some particles to be exposed to lower or high temperatures depending on the path of the particles, resulting in some unconverted $\mathrm{CuO}$ found in the treated product.

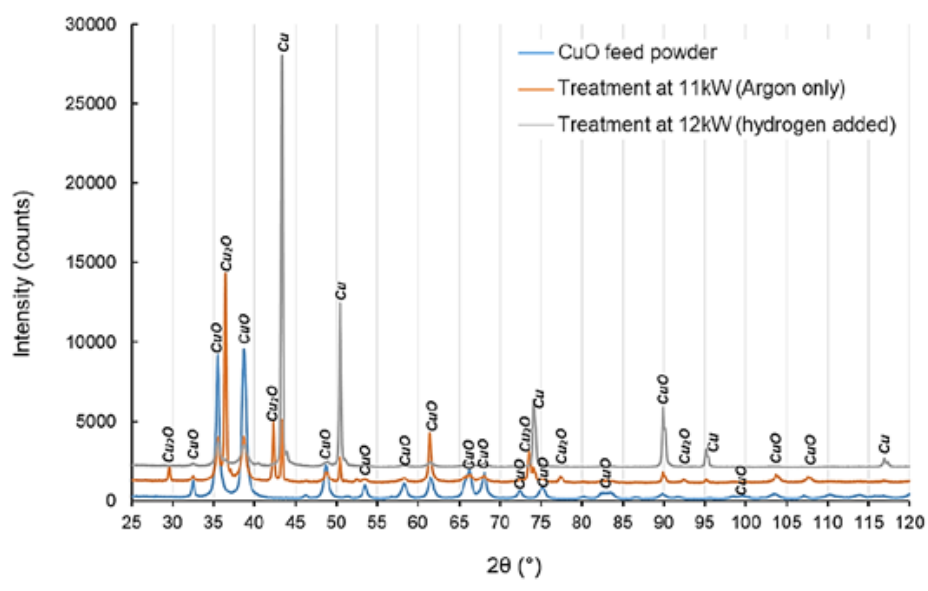

Figure 2: XRD spectra of the copper(II) oxide feed powder, the powder treated at $11 \mathrm{~kW}$ in argon only and the powder treated at $12 \mathrm{~kW}$ with the addition of hydrogen 
Table 2: Proposed phase proportions as determined by Rietveld refinement of the copper(II) oxide feed and plasma treated powders as determined from XRD analysis

\begin{tabular}{ccccc}
\hline Plasma plate power & $\mathbf{H}_{\mathbf{2}}$ in sheath gas & \multicolumn{3}{c}{ Proposed phases (\%) } \\
\hline $\mathbf{( k W )}$ & $\mathbf{( v / \mathbf { v } \% )}$ & $\mathbf{C u O}$ & $\mathbf{C u}_{\mathbf{2}} \mathbf{O}$ & $\mathbf{C u}$ \\
\hline $\mathrm{CuO}$ feed & - & 100 & 0 & 0 \\
11 & 0 & 25 & 59 & 16 \\
12 & 5 & 14 & 0 & 86 \\
\hline
\end{tabular}

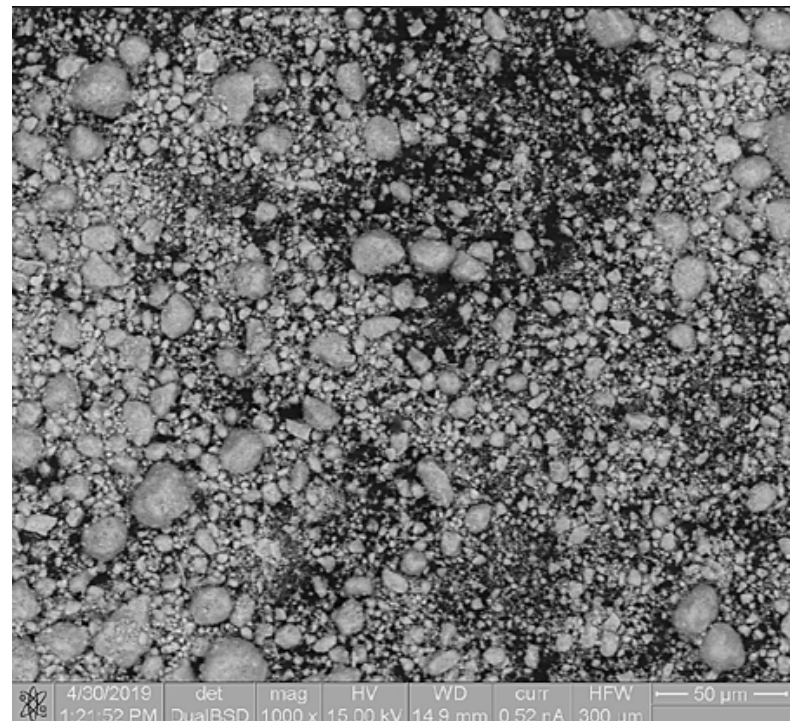

Figure 3: The SEM image of the copper(II) oxide powder as supplied by Merck. This powder was used as feed powder for experiments

The SEM images of all the powders are shown in Figure 3 - Figure 5. From the SEM images it could be seen that the feed powder contained fine particles with agglomerates (Figure 3 ).

Although fine particles where still predominately present after plasma treatment at $11 \mathrm{~kW}$ using argon only, the formation of larger agglomerates could be seen (Figure 4 ).

The formation of more dense spherical particles was observed after plasma treatment at $12 \mathrm{~kW}$ in the presence of hydrogen (Figure 5 ), which could be attributed to melting of the material at higher plasma temperatures.

The EDS results are shown in Table 3. It is difficult and unreliable to quantify the light element composition of the powder using the EDS technique, this being a known limitation of X-ray based elemental quantification.

However, there is a clear difference in the EDS results with seemingly less oxygen present in the sample treated at $12 \mathrm{~kW}$ with hydrogen with 93 atomic \% of copper measured. These observations

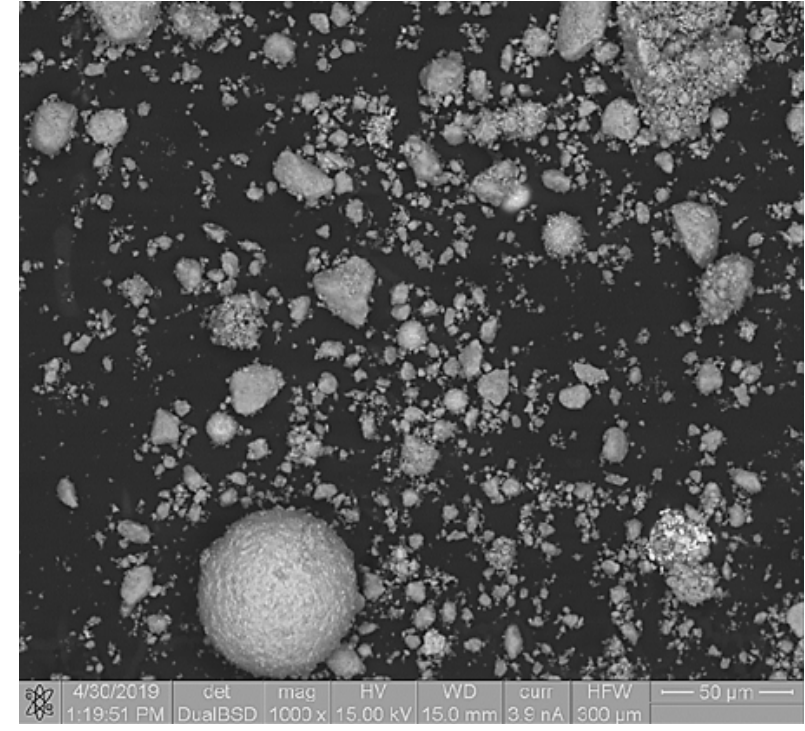

Figure 4: The SEM image of the powder collected after the plasma treatment at $11 \mathrm{~kW}$ in argon only

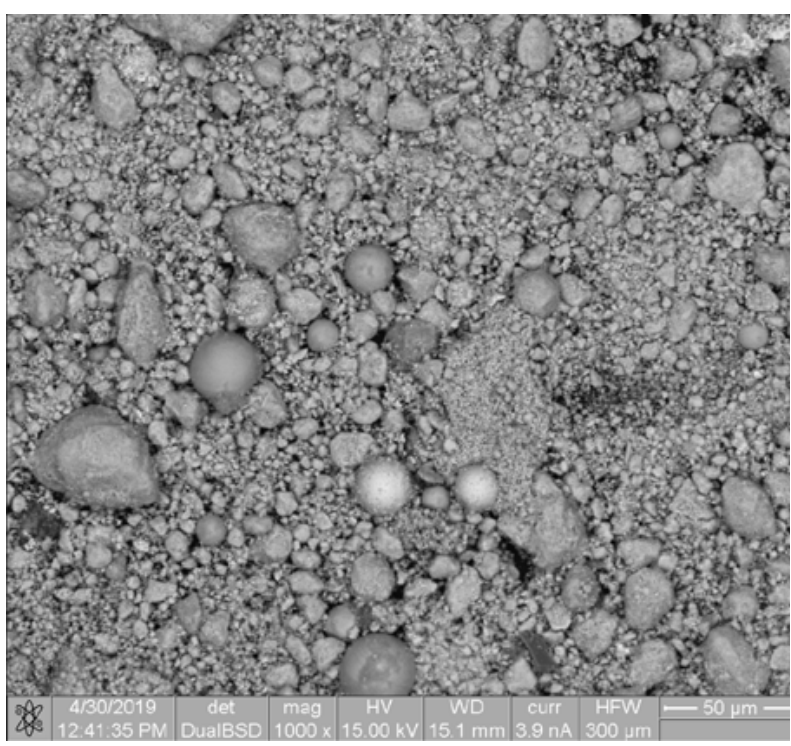

Figure 5: The SEM image of the powder collected after the plasma treatment at $12 \mathrm{~kW}$ with hydrogen gas $(5 \mathrm{v} / \mathrm{v} \%)$

Table 3: Quantities of $\mathrm{Cu}$ and $\mathrm{O}$ in the copper(II) oxide feed and plasma treated powders as determined from EDS analysis

\begin{tabular}{cccc}
\hline \multirow{2}{*}{ Plasma plate power } & \multirow{2}{*}{ in sheath gas } & \multicolumn{2}{c}{ Atomic \% } \\
\cline { 3 - 4 } & & $\mathbf{C u}$ & $\mathbf{O}$ \\
\hline $\mathrm{kW})$ & $\mathbf{( v / \mathbf { v } \% )}$ & $\mathbf{( \% )}$ & $41 \pm \mathbf{1 0 . 1 8}$ \\
\hline 11 & 0 & $59 \pm \mathbf{4 . 4 4}$ & $62 \pm \mathbf{8 . 7 0}$ \\
12 & 5 & $38 \pm \mathbf{4 . 4 4}$ & $7 \pm \mathbf{9 . 7 8}$ \\
\hline
\end{tabular}




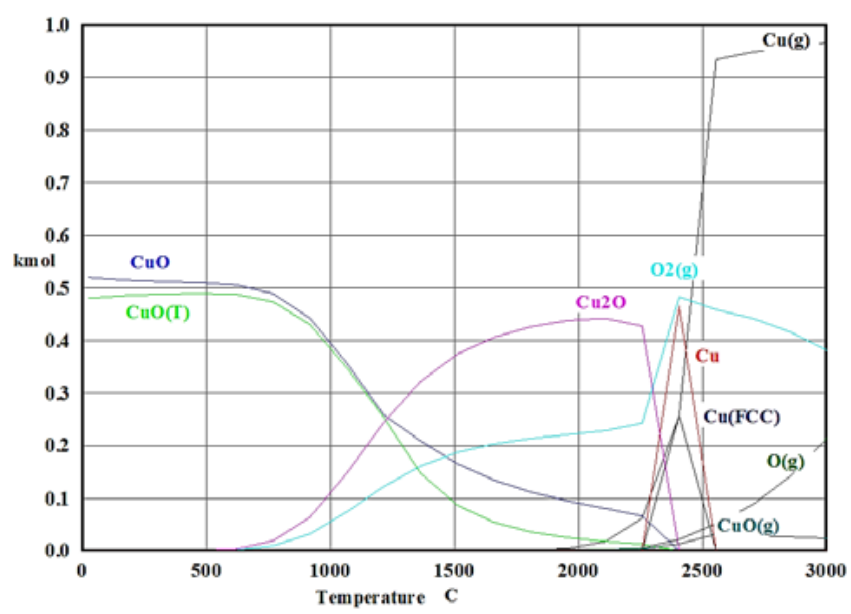

Figure 6: Equilibrium compositions of thermally reducing copper(II) oxide in argon only at temperatures ranging from 0 to $3000{ }^{\circ} \mathrm{C}$

supported the XRD analysis results discussed earlier. The EDS results indicated that the oxygen content increased after plasma treatment at $11 \mathrm{~kW}$ without the presence of hydrogen. The possible cause of this result might be the migration of oxygen to the surface of the particles due to high temperature treatment of the powder. EDS is a surface analysis method and therefore crosssectional particle analysis might have given a clearer indication of the $\mathrm{Cu}$ and $\mathrm{O}$ quantities present in the particles.

The results of the thermodynamic modelling of the reduction of copper(II) oxide, with and without hydrogen, are shown in Figure 6 - Figure 8 . Figure 6 shows the results of thermally reducing $1 \mathrm{kmol}$ of copper(II) oxide. Noticeable thermal reduction of copper(II) oxide was observed above $500{ }^{\circ} \mathrm{C}$. Copper(II) $(\mathrm{CuO})$ was reduced to copper $(\mathrm{I})\left(\mathrm{Cu}_{2} \mathrm{O}\right)$ instead of $\mathrm{Cu}$ metal. The formation of copper vapour was observed at $c a .2000{ }^{\circ} \mathrm{C}$ from the reduction of either copper(II) oxide or copper(I) oxide. There was a sharp increase in the formation of copper vapour at ca. $2250^{\circ} \mathrm{C}$ and liquid copper started to form at the same temperature. A sharp decrease in copper(I) oxide was also observed at $c a .2250{ }^{\circ} \mathrm{C}$. The equilibrium compositions of thermally reducing copper(II) oxide suggests that for successful reduction of copper(II) oxide to copper, the copper(II) oxide particle must absorb enough energy from the

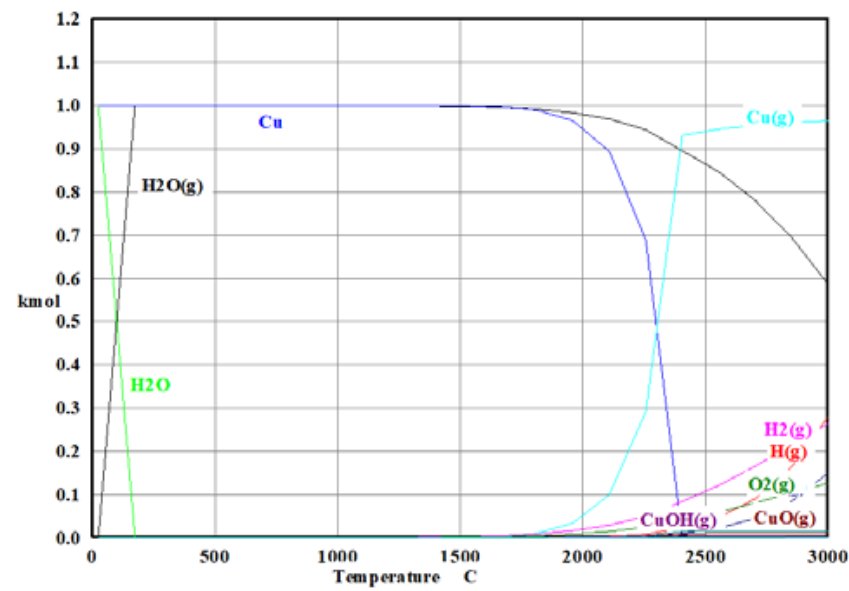

Figure 7: Equilibrium compositions of thermally reducing copper(II) oxide in the presence of $1 \mathrm{kmol}$ hydrogen at temperatures ranging from 0 to $3000{ }^{\circ} \mathrm{C}$

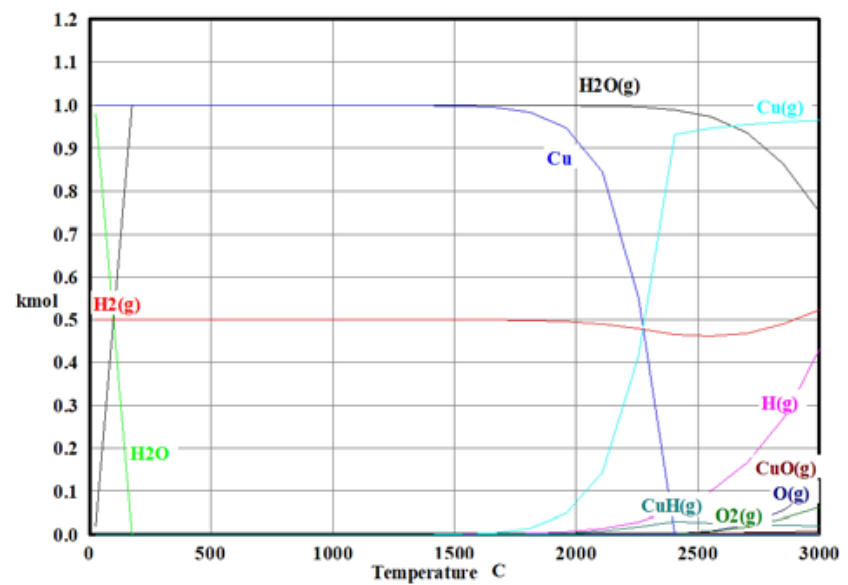

Figure 8: Equilibrium composition of reducing copper(II) oxide thermally in the presence of $1.5 \mathrm{kmol}$ hydrogen at temperatures ranging from 0 to $3000{ }^{\circ} \mathrm{C}$

plasma to reach temperatures greater than $2400{ }^{\circ} \mathrm{C}$. Most of the oxides were reduced to copper above $2400{ }^{\circ} \mathrm{C}$, and only a trace of copper(II) oxide remained. These results indicated that the thermal argon plasma obtained at $11 \mathrm{~kW}$ did not heat the copper(II) oxide particles to a temperature greater than $2400{ }^{\circ} \mathrm{C}$ and therefore only a limited amount of copper(II) oxide was converted to copper metal.

Figure 7 and Figure 8 show the results of using $1 \mathrm{kmol}$ of copper(II) oxide with 1 and $1.5 \mathrm{kmol}$ of hydrogen gas, respectively.

The program predicted that copper(II) oxide would be reduced directly to copper at a temperature higher than $2000{ }^{\circ} \mathrm{C}$ when stoichiometric or greater amounts of hydrogen were used. The reduction of copper(II) oxide to copper, in the presence of stoichiometric or greater amounts of hydrogen, required less energy to achieve compared to using sub-stoichiometric amounts of hydrogen or without hydrogen entirely. This is likely due to the formation of water, which has a lower free energy than diatomic oxygen and hydrogen gases. This thermodynamically drives the reaction towards producing copper metal and water using less added energy than is required to produce copper metal and diatomic oxygen gas. ${ }^{10}$ The requirement for stoichiometric quantities of hydrogen adds credence to this conjecture as the hydrogen would likely be functioning as an oxygen scavenger, and not catalytically.

The thermodynamic study using HSC Chemistry 6 software predicted that the reduction of copper(II) oxide was favourable thermally with and without hydrogen gas. However, the program predicted that thermal reduction, either without or with substoichiometric amounts of hydrogen might require more energy. When stoichiometric or excess hydrogen is used the program predicted that copper(II) oxide would be reduced directly to copper, requiring less energy. The reduction of $\mathrm{CuO}$ to metallic $\mathrm{Cu}$ without the formation of intermediates or suboxides has been observed in a study by Kim et al. ${ }^{10}$ These results were in agreement with the plasma treatment of copper(II) oxide in the presence of hydrogen. This is in agreement with a study conducted where $\mathrm{CuCO}_{3}$ was reduced to $\mathrm{Cu}$ nano-particles in a microwave plasma. The precursor chemically reacts with the plasma species in the gas phase. ${ }^{12}$ In addition, the HSC software does not take kinetics into account, so the improved thermal transfer properties of a hydrogen-containing plasma would not impact these calculations. The improved thermal 
conductivity of the hydrogen-containing plasma would enable particles to reach higher temperatures faster, further improving the reducing ability of this gas mixture.

\section{Conclusion}

The addition of copper powder during the additive manufacturing process used to produce $3 \mathrm{D}$ printed medical implants may yield titanium implants with antibacterial functionality. A possible method to achieve this is to make use of a thermal plasma to convert various copper oxide powders to copper metal.

In order to evaluate whether copper oxide can be reduced to copper metal making use of a thermal plasma, the Tek-15 thermal plasma system was utilised and the thermal treatment of copper(II) oxide powder investigated with and without the addition of hydrogen. The black copper(II) oxide powder was converted to a yellow / orange colour without hydrogen being present and a dark red powder with hydrogen present in the plasma. XRD results confirmed that copper(II) oxide was converted to copper metal in a plasma containing hydrogen, while mostly copper(I) oxide was obtained in the absence of hydrogen, under the tested conditions. Thermodynamic calculations indicated that $\mathrm{Cu}$ metal will be produced when copper(II) oxide is plasma treated in the presence of hydrogen. The study indicated that fine copper powder could be produced using thermal plasma technology. This technique can be used to prepare other metals and metal alloys with these powders being useful in different fields. ${ }^{12}$

\section{Acknowledgements}

The authors would like to acknowledge the Department of Science and Innovation (DSI), through the Advanced Materials Initiative (AMI), for the financial support in conducting this study. The
South African Nuclear Energy Corporation SOC Ltd. (Necsa) is acknowledged for their financial support. Dr. Tshepo Ntsoane from Necsa is hereby thanked for the XRD results.

\section{References}

1. ASTM, 2012. ASTM F2792e12a: Standard Terminology for Additive Manufacturing Technologies. ASTM International, West Conshohocken, PA.

2. Ford, S., Despeisse, M., 2016. Additive manufacturing and sustainability: an exploratory study of the advantages and challenges. Journal of Cleaner Production 137, 1573-1587.

3. Zhong, C., Liu, J., Zhao, T., Schopphoven, T., Fu, J., Gasser, A., Schleifenbaum, J.H., 2020. Laser Metal Deposition of Ti6A14V - A Brief Review. Applied Sciences 10, 764

4. Geetha, M., Singh, A.K., Asokamani, R., Gogia, A.K., 2009. Ti based biomaterials, the ultimate choice for orthopaedic implants - A review. Progress in Materials Science 54, 397-425.

5. Romanò, C.L., Scarponi, S., Gallazzi, E., Romanò, D., Drago, L., 2015. Antibacterial coating of implants in orthopaedics and trauma: a classification proposal in an evolving panorama. J Orthop Surg Res $10,157$.

6. Krakhmalev, P., Yadroitsev, I., Yadroitsava, I., de Smidt, O., 2017. Functionalization of Biomedical Ti6A14V via In Situ Alloying by $\mathrm{Cu}$ during Laser Powder Bed Fusion Manufacturing. Materials 10, 1154.

7. Vilardell, A.M., Yadroitsev, I., Yadroitsava, I., Albu, M., Takata, N., Kobashi, M., Krakhmalev, P., Kouprianoff, D., Kothleitner, G., Plessis, A. du, 2020. Manufacturing and characterization of in-situ alloyed Ti6Al4V(ELI)-3 at.\% Cu by laser powder bed fusion. Additive Manufacturing 36, 101436

8. Andal, V., Buvaneswari, G., 2017. Effect of reducing agents in the conversion of $\mathrm{Cu}_{2} \mathrm{O}$ nanocolloid to $\mathrm{Cu}$ nanocolloid. Engineering Science and Technology, an International Journal 20, 340-344.

9. Wahyudi, S., Soepriyanto, S., Mubarok, M.Z., Sutarno, 2018. Synthesis and Applications of Copper Nanopowder - A Review. IOP Conf. Ser.: Mater. Sci. Eng. 395, 012014.

10. Kim, J.Y., Rodriguez, J.A., Hanson, J.C., Frenkel, A.I., Lee, P.L., 2003. Reduction of $\mathrm{CuO}$ and $\mathrm{Cu}_{2} \mathrm{O}$ with $\mathrm{H}_{2}: \mathrm{H}$ Embedding and Kinetic Effects in the Formation of Sub-oxides. J. Am. Chem. Soc. 125, 10684-10692

11. Markina, N.E., Pozharov, M.V., Markin, A.V., 2016. Synthesis of Copper (I) Oxide Particles with Variable Color: Demonstrating Size-Dependent Optical Properties for High School Students. J. Chem. Educ. 93, 704-707.

12. Chau, J., Yang, C.-C., Shih, H.-H., 2014. Microwave Plasma Production of Metal Nanopowders. Inorganics 2, 278-290. 